International Conference on New Interfaces for Musical Expression

\title{
Diastema for the time \\ floating between the networked remote places
}

Mikako MIZUNO ${ }^{1}$, Yoshihisa SUZUKI ${ }^{2}$

${ }^{1}$ Nagoya Clty University, ${ }^{2}$ Nagoya University of Arts and Sciences

Published on: May 24, 2021

License: Creative Commons Attribution 4.0 International License (CC-BY 4.0). 


\section{PROJECT DESCRIPTION}

Diastema in Ancient Greek means a space between two adjacent substances and can be explained as interval. In the Western Middle Ages the term indicates an adjective describing notation.

In my music series Diastema, two pianos were set in two different venues and were connected through network. MIDI signals were sent on OSC and the Disklavier was interactively played by the pianist's performance which occurred in the remote place. Audio information was transmitted through DANTE or Jacktrip in order to get highspeed response.

The short edition of the first performance (2020 Feb.22, Nagoya, Japan) can be found in Vimeo.

\section{https://vimeo.com/510599956}

For NIME2021, I present a new version of "Diastema", that is ,"Diastema II" which will be performed in two remote places. The two places will audio-visually connected. Because of several layers of latency (LAN, audio processing, hardware transmission, etc.), the audience will not only see the time difference between the two but also hear the tuning difference between the instruments. Interval of the pitches, separation of the places and time difference are the three dimension of the piece. The audience can see and hear all the three dimensions from his/her own point. Where and when is the point?

\section{PROGRAM NOTES}

In the first performance of this piece, two pianos were set in two different buildings and were connected through network. One piano was tuned in $440 \mathrm{hz}$ and the other in 442hz. MIDI signals were sent on OSC and the Disklavier was interactively played by the pianist's performance. Audio information was transmitted through DANTE in order to get high-speed response. "Diastema II"is performed in two remote places. The two places are audio-visually connected. Because of several layers of latency (LAN, audio processing, hardware transmission, etc.), the audience will not only see the time difference between the two but also hear the tuning difference between the instruments. Interval of the pitches, separation of the places and time difference are the three dimension of the piece. The audience can see and hear all the three dimensions from an objective point. 


\section{PERFORMANCE REQUIREMENTS}

- equipment: Zoom or the other video conference application to receive our online performance. The live image and sound of the zoom should be projected to the audience.

- space: Space for presenting this piece, a traditional concert stage with 2 ch speakers and a visual projector will be suitable. Other public space is possible if that has $2 \mathrm{ch}$ speakers and a visual projector.

- performer(s): Our networked performance will be set up in OUR places including the performers. All the performers are in several separated places but all the performers will be managed in OUR places.

- feasibility: Our team has 7-year experiences to perform the networked music, including ICMC,ISCM,NEMC, JSSA etc. See below;

https://www.academia.edu/4823023/Asian_Network_Music_Performances_with_Jacktrip

\section{MEDIA}



Fig.1 Disklavier in the NCU studio 




Fig.2 left: Percussionist controls disklavier right: Pianist controls two pianos



Fig.3 A networked performance between Taipei and Nagoya 